\title{
HEPATITIS CRÓNICAS VIRALES B y C EN POBLACIÓN INMIGRANTE EN ESPAÑA
}

\author{
Enrique Calderón Sandubete (1), Rosa Yang Lai (1), María de la Luz Calero Bernal (2), María Teresa \\ Martínez Rísquez (2), María Calderón Baturone (3) y Carmen de la Horra Padilla (1).
}

(1) CIBER de Epidemiología y Salud Pública e Instituto de Biomedicina de Sevilla. Hospital Universitario Virgen del Rocío/CSIC/Universidad de Sevilla., Sevilla

(2) Servicio de Medicina Interna. Hospital Universitario Virgen de Rocío. Sevilla .

(3) Unidad Docente de Medicina Familiar y Comunitaria. Hospital Universitario de Valme. Sevilla.

Los autores no tienen conflictos de interés en relación con los contenidos de este artículo.

\section{RESUMEN}

Fundamentos: En España la prevalencia de la hepatitis crónicas de origen viral puede variar a causa de los inmigrantes procedentes de áreas de elevada prevalencia de infección por virus $\mathrm{B}$ y $\mathrm{C}$ de la hepatitis. La infección por estos virus es un problema importante de salud pública global por los procesos crónicos que originan. El objetivo del estudio fue conocer el impacto de la inmigración en la prevalencia de las hepatitis crónicas virales en España.

Métodos: Revisión bibliográfica cualitativa de la literatura científica sobre el tema publicada entre enero de 1998 y diciembre de 2012 utilizando las bases Medline y MEDES-MEDicina.

Resultados: Se analizaron los datos procedentes de 19 artículos originales. En conjunto la prevalencia de infección por los virus $\mathrm{B}$ y C de la hepatitis fue mayor en la población emigrante que la descrita para la población general española. Los emigrantes de África y Europa del Este presentaron las mayores prevalencias y los inmigrantes iberoamericanos las menores.

Conclusiones: La prevalencia de las infecciones por virus $\mathrm{B}$ y $\mathrm{C}$ de la hepatitis en inmigrantes sugiere que podrían tener un importante impacto en la salud pública en España.

Palabras clave: Virus Hepatitis B. Hepatitis Crónica B. Virus Hepatitis C. Hepatitis Crónica C. Emigrantes. Inmigrantes. España.

\section{Correspondencia}

Enrique J. Calderón

Instituto de Biomedicina de Sevilla

Hospital Universitario Virgen del Rocío

Avda. Manuel Siurot s/n, 41013 Sevilla

Spain

sandube@cica.es

\section{ABSTRACT}

\section{Chronic Viral Hepatitis B and C in Immigrat population, Spain}

Background: the prevalence of chronic viral hepatitis in Spain could vary because of the immigrants coming from countries having an elevated with a higher endemicity of hepatitis B and C virus. Hepatitis B and hepatitis $\mathrm{C}$ virus infections are an important health problem worldwide taking into account their chronic consequences. The aim of this study was to know the impact of immigration in the prevalence of chronic viral hepatitis in Spain.

Methods: qualitative of scientific papers searching in Medline and MEDES-MEDicina, with date limit January 1998- December 2012 and only papers in English and Spanish.

Results: data from 19 original articles were analyzed. The prevalences of hepatitis B and C virus infections in the immigrant population, on the whole, are higher than Spanish population. Immigrants from Africa and East European countries presented the higher prevalence of Hepatitis B and Hepatitis $\mathrm{C}$ virus infection, whereas the Latin American-origin population displayed the lowest one.

Conclusion: the prevalences of hepatitis B and C virus infections in the immigrant population suggest they could have a substantial public health impact in Spain.

Keyword: Hepatitis B virus. Hepatitis B, chronic. Hepacivirus. Hepatitis C, chronic. Emigrants. Immigrants. Spain. 


\section{INTRODUCCIÓN}

Actualmente las infecciones por los virus B (VHB) y C (VHC) de la hepatitis constituyen un problema de salud púbica de alcance mundial con un gran impacto también en nuestro país, fundamentalmente debido a que en conjunto son la causa más común de cirrosis hepática y hepatocarcinoma ${ }^{1}$.

Se calcula que el VHB ha podido infectar a casi un tercio de la población mundial y que unos 150 millones de personas sufren la forma crónica de la infección. Su distribución geográfica varía mucho, identificándose tres áreas según la prevalencia de la infección crónica por el VHB sea alta $(>8 \%)$, intermedia $(2-7 \%)$ o baja $(<2 \%)$. Entre las áreas de alta prevalencia se incluyen Europa oriental, el sudeste asiático, China y África subsahariana. En España el porcentaje de portadores crónicos se acerca al $1 \%$ de la población y un $30 \%$ son infecciones activas que tienen un elevado riesgo de progresar hacia cirrosis hepática y hepatocarcinoma ${ }^{2,3}$.

La infección por VHC es cosmopolita afectando aproximadamente al $3 \%$ de la población mundial. Su prevalencia en la población general varía entre el 0,2 y el $18 \%$ según la zona geográfica considerada. Las áreas de mayor prevalencia incluyen los países del Lejano Oriente, la cuenca mediterránea y ciertas áreas de África y Europa Oriental $^{2,3}$. En nuestro medio el 2,5\% de la población presenta una infección crónica por VHC, causa principal de hepatitis crónica, cirrosis y carcinoma hepatocelular, representando la mayor indicación de trasplante hepático. En conjunto las infecciones crónicas por los virus $\mathrm{B}$ y $\mathrm{C}$ de la hepatitis son responsables anualmente de la muerte de alrededor de un millón de personas en todo el mundo y juntas afectan a una de cada 12 personas $^{2}$.

La inmigración se ha convertido en un fenómeno de creciente importancia en nues- tro país, habiéndose multiplicado en la última década por tres el número de extranjeros residentes en España, alcanzando los 5,7 millones de habitantes que representa más del $12 \%$ del total de nuestra población. España es el segundo país de la Unión Europea con más población extranjera y donde ésta representa una de las proporciones más altas respecto de la población total ${ }^{4}$.

En estas circunstancias cabe esperar que la prevalencia de las hepatitis crónicas víricas en España pueda variar debido a la recepción de inmigrantes procedentes de países con elevada endemia de infección crónica por VHB y VHC. Los estudios sobre morbilidad en población extranjera se han centrado sobre todo en enfermedades importadas y solo recientemente se ha comenzado a analizar las enfermedades crónicas, pero dado el gran impacto sanitario y económico que puede tener el incremento progresivo de pacientes con hepatitis crónica de origen viral es necesario disponer de datos que nos aproximen al conocimiento de la situación real de la infección por VHB y $\mathrm{VHC}$ en estos grupos poblacionales para poder planificar estrategias específicas de detección, tratamiento y prevención ${ }^{5,6}$.

El objetivo de este estudio es conocer la prevalencia de infecciones crónicas por virus $\mathrm{B}$ y $\mathrm{C}$ de la hepatitis en la población inmigrante en nuestro país.

\section{MATERIAL Y MÉTODOS}

Se realizó un estudio de revisión de la literatura científica sobre infección por los virus de la hepatitis $\mathrm{B}$ y $\mathrm{C}$ en la población inmigrante en España. Se llevó a cabo en el marco de una revisión más amplia realizada a nivel nacional y con la participación de las líneas de investigación del Subprograma de Inmigración y Salud del CIBERESP.

Para la búsqueda de artículos científicos publicados entre enero 1998 y diciembre 2012 se consultaron las bases de datos de 
Medline/Pubmed y MEDES-MEDicina en español, debido a su mayor visibilidad internacional y nacional, respectivamente, en el área sanitaria. La decisión de limitar la búsqueda al período establecido se basó en la intención de explorar toda la producción científica generada sobre este tema desde el inicio del incremento progresivo de la llegada de población inmigrante a España.

En la base de datos con descriptores o vocabulario controlado (tesauros) como Medline, se emplearon los Medical Subject Headings [MeSH] para realizar la búsqueda. Para la ecuación de búsqueda, se desarrollaron 3 filtros temáticos: a) Filtro sobre el tema de revisión: Hepatitis B virus; Hepatitis B, Chronic; Hepacivirus; Hepatitis C, Chronic; b) Filtro inmigración: Emigrants and Immigrants, Emigration and Immigration, Transients and Migrants y Ethnic Groups; c) Filtro país: se utilizó el desarrollado por Valderas y colaboradores ${ }^{7}$. El término limitador por contenido fue la especie humana. En cuanto al idioma, se seleccionaron las publicaciones escritas en español e inglés.

En la base de datos MEDES-MEDicina en español, que no cuenta con tesauro, se utilizaron las siguientes palabras: "migración", "migrantes", "migrante", "inmigración", "inmigrantes", "inmigrante". Los operadores boléanos "AND" y "OR" fueron utilizados para recuperar toda la literatura existente en ambas bases de datos.

Para la selección de los artículos se estableció como criterios de inclusión artículos originales sobre infección por los virus B y $\mathrm{C}$ de la hepatitis en la población inmigrante procedente de Iberoamérica, África, Asia y de Europa del Este, que hayan sido desarrollados en España. Además, estudios que aunque no especificaran la nacionalidad de los inmigrantes incluyeran las categorías de inmigrantes, extranjeros o similares que respondieran a la definición de inmigrante otorgada por la Organización Internacional de Migraciones (OIM $)^{8}$. Se excluyeron los estudios realizados en población inmigrante proveniente de países diferentes a los señalados anteriormente y otros tipos de publicaciones como editoriales, cartas al director, revisiones teóricas o sistemáticas y comunicaciones a congresos.

El proceso de selección de los estudios se realizó en 3 fases -cribado, revisión y elegibilidad-. Para la extracción de los datos se elaboró un protocolo de recogida de información para estudios cuantitativos y cualitativos. Posteriormente, se digitalizó su contenido a través de la herramienta Google DRIVE al que puede accederse a través del siguiente vínculo http://goo.gl/PU2fvs.

\section{RESULTADOS}

Se identificaron 19 artículos que cumplían los criterios establecidos y abordaban la prevalencia de las infecciones crónicas por virus $\mathrm{B}$ y $\mathrm{C}$ de la hepatitis en la población inmigrante residente en España. El ámbito de realización de los estudios y la población analizada fue muy variable de unos a otros.

Uno de los estudios analizó sólo la población de niños inmigrantes menores de 14 años atendida en una Unidad de Medicina Tropical entre 1989 y $2001^{9}$. De 75 niños africanos 5 eran portadores del antígeno de superficie del VHB $(6,6 \%)$ y uno de 6 asiáticos estudiados $(16,6 \%)$. No se encontraron portadores de VHB entre los 27 niños iberoamericanos analizados. Por otra parte, sólo se encontró infección por VHC en el 1,7\% de los niños africanos estudiados y en ninguno de los procedentes de Asia o Iberoamérica? .

Otros dos estudios analizaron específicamente la situación de mujeres inmigrantes embarazadas ${ }^{10,11}$. En uno de ellos se encontró el $1 \%$ de infección por VHC entre las gestantes españolas y el $0,9 \%$ entre las inmigrantes consideradas globalmente, aunque la prevalencia era mayor en las pro- 
Figura 1

Proceso de selección de los artículos incluidos en la revisión bibliográfica

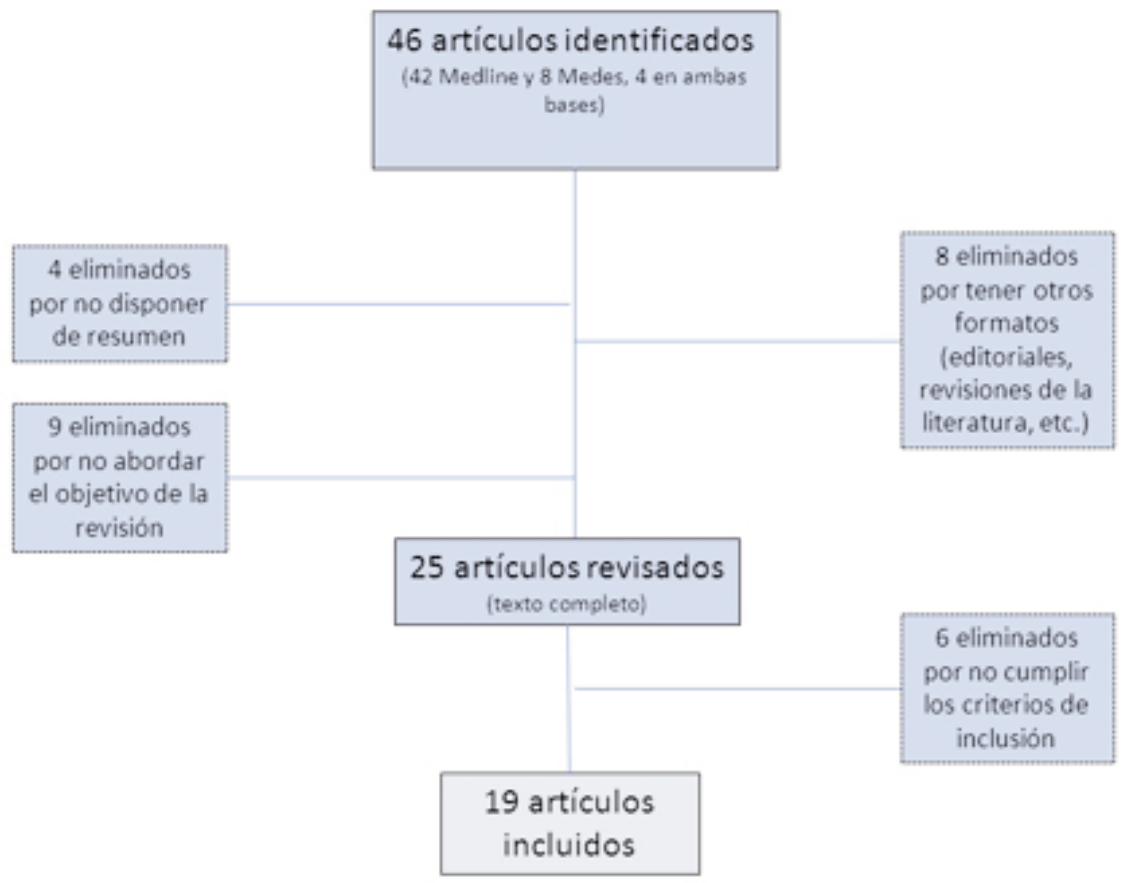

Tabla 1

Prevalencia de infección por virus de la hepatitis B según las zonas de procedencia de la población inmigrante

\begin{tabular}{|c|c|c|c|c|c|}
\hline Autor & Ámbito del estudio & Latinoamérica & África & Asia & Europa Este \\
\hline López-Vélez, 2003 & Madrid & 0 & 9 & 0 & 20 \\
\hline Ramos, 2003 & Elche & 0 & 2,5 & - & 2,1 \\
\hline Toro, 2006 & Madrid & 0 & 8 & - & 0 \\
\hline Manzardo, 2008 & Barcelona & 0,6 & 12 & 1,9 & 2,7 \\
\hline Valerio, 2008 & Cataluña & 1,2 & 18,2 & $\begin{array}{c}7,1 \\
5,5 \text { (India) }\end{array}$ & 13,1 \\
\hline Monge-Maillo, 2009 & Madrid & - & 9,8 & - & - \\
\hline Pérez Molina, 2009 & $\begin{array}{c}\text { Madrid } \\
\text { (pacientes con infección VIH) }\end{array}$ & 0 & 11,1 & - & - \\
\hline Caro-Murillo, 2009 & $\begin{array}{c}\text { Madrid } \\
\text { (pacientes con infección VIH) }\end{array}$ & 4 & 8,9 & - & 7,1 \\
\hline Salas, 2011 & Almería & - & 26,9 & - & - \\
\hline Ramos, 2011 & Elche & 2,4 & 12,8 & 35,3 & 16,7 \\
\hline Pérez-Molina, 2011 & Madrid & 3,6 & 13,3 & - & - \\
\hline
\end{tabular}


Tabla 2

Prevalencia de infección por virus de la hepatitis $\mathrm{C}$ según las zonas de procedencia de la población inmigrante

\begin{tabular}{|l|c|c|c|c|c|}
\hline \multicolumn{1}{|c|}{ Autor } & Ámbito del estudio & Latinoamérica & África & Asia & Europa Este \\
\hline López-Vélez, 2003 & Madrid & 0 & 10,5 & 15,8 & 0 \\
\hline Ramos, 2003 & Elche & 0,4 & 1,9 & - & 1 \\
\hline Toro, 2006 & Madrid & 0,9 & 4,9 & - & 5,6 \\
\hline Manzardo, 2008 & Barcelona & 0,6 & 3,5 & 4,6 & 5,7 \\
\hline Valerio, 2008 & Cataluña & 1,4 & 3,5 & 0 & 14,9 (India) \\
\hline Pérez Molina, 2009 & $\begin{array}{c}\text { Madrid } \\
\text { (pacientes con infección VIH) }\end{array}$ & 0 & 9,8 & - & - \\
\hline Caro-Murillo, 2009 & $\begin{array}{c}\text { Madrid } \\
\text { (pacientes con infección VIH) }\end{array}$ & 5,6 & 11,7 & - & 38 \\
\hline Ramos, 2011 & Elche & 0,7 & 1,5 & 0 & 23,4 \\
\hline Salas, 2011 & Almería & - & 5,9 & - & - \\
\hline
\end{tabular}

cedentes de determinados países como Rumanía, que alcanzaba el $4,1 \%{ }^{10}$. En este mismo estudio se detectó antígeno de superficie del VHB en el $1,1 \%$ de las mujeres embarazadas españolas y en el $2 \%$ de las extranjeras, presentando las mayores tasas las de origen asiático con un 10,9\% y las procedentes de Europa del Este con un $4,2 \%{ }^{10}$. Datos similares a los hallados en otro estudio donde también la prevalencia de mujeres gestantes portadoras de VHB fue mayor en inmigrantes $(2,6 \%)$ que en españolas $(0,4 \%)$, correspondiendo la mayor diferencia de prevalencia a la población nativa a las mujeres gestantes asiáticas (OR=18,6; IC del 95\%, 5,2-66,3) y las procedentes de Europa del Este (OR=16; IC del $95 \%, 5,7-44,4)^{11}$.

En sendos estudios realizados entre mujeres inmigrantes que ejercían la prostitución en Madrid se identificó una tasa similar de infección por $\mathrm{VHC}$ con el $0,8 \%$ y el $0,93 \%$ (IC 95\%: 0,34-2,30) respectivamente. Sin embargo, la tasa de portadoras de VHB fue diferente con un 3,5\% de portadoras de VHB en un caso y el 0,52\% (IC 95\%: 0,13$1,64)$ en el otro, probablemente debido a que en el primer estudio predominaban las mujeres inmigrantes de origen subsahariano y en el segundo de Iberoamérica ${ }^{12,13}$.
Seis artículos correspondían a estudios realizados en pacientes inmigrantes atendidos en Unidades Especializadas en Medicina Tropical ${ }^{14-19}$, otros seis eran estudios realizados en consultas de enfermedades infecciosas $^{20-25}$ y dos en el ámbito de la Asistencia Primaria y en un centro de la Cruz Roja $^{26,27}$. Las diferentes prevalencias encontradas para las infecciones por VHB y VHC según las zonas de procedencia de la población inmigrante se recogen en las tablas 1 y 2 .

En uno de los estudios realizados en una consulta de enfermedades de transmisión sexual la frecuencia de infección por VIH fue similar en la población inmigrante y en la autóctona (1,8 y $1,7 \%$ respectivamente), mientras que los portadores de VHB fueron algo más frecuentes entre los inmigrantes $(2,6 \%)$ frente al $0,9 \%$ en los autóctonos ${ }^{20}$.

En otro estudio que incluyó a todos los pacientes atendidos por primera vez en una Unidad monográfica de VIH se encontró una diferencia estadísticamente significativa respecto a la presencia de infección por VHC entre la población inmigrante y la autóctona: $8,9 \%$ frente al $50,4 \%(\mathrm{p}<0,001)$, pero no así respecto a la de portadores de VHB $(8,9 \text { frente a } 11,3 \%, p=0,99)^{22}$. Por otra parte, en otro estudio que encontró una 
prevalencia de portadores de VHB del 7\% se describió la presencia de dos pacientes portadores de VHB con la mutación rtM204V vinculada a la resistencia a lamivudina ${ }^{24}$.

\section{DISCUSIÓN}

La información disponible en España sobre prevalencia de las infecciones crónicas por virus de la hepatitis $\mathrm{B}$ y $\mathrm{C}$ en la población inmigrante es escasa, sobre todo en grupos concretos que pueden tener especial interés epidemiológico, como los niños, las mujeres embarazadas o las trabajadoras del sexo ${ }^{9-13,28,29}$.

En general los datos disponibles indican que la frecuencia de individuos portadores crónicos de la infección por virus de la hepatitis B es elevada en inmigrantes procedentes de zonas consideradas de alta prevalencia, como Europa oriental, Asia o el África subsahariana, donde hay prevalencias superiores a la existente en la población general española. Lo mismo sucede con la infección por virus de la hepatitis $C$, que presenta las tasas más elevadas entre inmigrantes procedentes de zonas de mayor prevalencia, como ciertas áreas de África y Europa Oriental, mientras que las tasas de infección son, en general, menores que las registradas en la población española entre inmigrantes iberoamericanos $^{3}$.

En general, podemos considerar que los porcentajes de infección crónica por virus de la hepatitis B y C encontrados en los diferentes estudios publicados dependen tanto de las zonas de origen de los inmigrantes como de la procedencia institucional del colectivo estudiado. En este sentido, la principal limitación que encontramos a la hora de interpretar los datos obtenidos en los diferentes estudios es que la mayoría fueron realizados en Unidades de Medicina Tropical o de Enfermedades Infecciosas, por lo que existe un evidente sesgo que no permite extrapolar las prevalencias encontradas a la población total de inmigrantes.
La mayoría de las enfermedades infecciosas típicas de los inmigrantes no tienen impacto, en términos de salud pública, en el país de destino, ya que su transmisión es muy difícil, bien por la falta de vector o de hospedadores intermediarios ${ }^{30}$. Sin embargo, las hepatitis virales crónicas merecen una especial consideración tanto por la carga de enfermedad que supone en años de vida perdidos por discapacidad y muerte prematura como por su coste para el sistema sanitario ${ }^{1,6,31-33}$. En este sentido, debe considerarse el impacto que la población inmigrante pueda tener en el aumento de estas enfermedades en nuestro medio, como refleja un reciente estudio donde el $53 \%$ de los nuevos casos diagnosticados de hepatitis crónica B correspondían a personas inmigrantes ${ }^{34}$.

Por otra parte, hay que considerar también que estas infecciones pueden transmitirse por vía parenteral y sexual, aunque esta última raramente en el caso del virus de la hepatitis $\mathrm{C}^{3}$. El riego de transmisión por sangre infectada está controlado en el ámbito sanitario por el cribado en los bancos de sangre y el uso de las precauciones estándar en el uso de material sanitario. Sin embargo, persiste en el uso de drogas parenterales y también para el virus de la hepatitis B en las relaciones sexuales sin protección, circunstancia especialmente relevante en el ámbito de la prostitución donde muchas de las trabajadoras del sexo son inmigrantes procedentes de áreas de alta prevalencia de infección por virus de la hepatitis $\mathrm{B}^{12,13}$.

En conclusión, esta revisión pone de manifiesto el importante papel que la inmigración puede tener en el incremento de la frecuencia de las infecciones crónicas por los virus $\mathrm{B}$ y $\mathrm{C}$ de la hepatitis así como en sus principales complicaciones, la cirrosis hepática y el hepatocarcinoma, con la consiguiente repercusión para el sistema sanitario. Las autoridades sanitarias de las comunidades con elevada proporción de 
inmigrantes deben estar preparadas para un incremento del gasto sanitario que puede ser sustancial, derivado de la atención sanitaria, tanto desde el punto de vista farmacéutico como de asistencia a las complicaciones de las hepatitis crónicas virales.

Es necesario un mejor conocimiento de la prevalencia de las hepatitis crónicas en la población inmigrante que vive en España y de las barreras que pueden tener para acceder a los cuidados sanitarios necesarios para controlar y reducir las infecciones por VHB y $\mathrm{VHC}$ en este colectivo vulnerable. Los inmigrantes procedentes de áreas de alta prevalencia de hepatitis crónica $\mathrm{B}$ y $\mathrm{C}$ deberían considerarse colectivo prioritario de intervención y monitorización, siendo primordial desarrollar campañas sanitarias dirigidas a estas comunidades de inmigrantes con el fin de evitar la transmisión de estos virus entre los miembros de las propias comunidades y la población autóctona.

\section{BIBLIOGRAFÍA}

1. García-Fulgueiras A, García-Pina R, Morant C, García-Ortuzar V, Génova R, Alvarez E. Hepatitis C and hepatitis B-related mortality in Spain. Eur J Gastroenterol Hepatol. 2009; 21: 895-901.

2. Rojo Marcos G, Cuadros González J, Arranz Caso A. Enfermedades infecciosas importadas en España. Med Clin 2008; 131: 540-50.

3. Aguilera Guirao A, Romero Yuste S y Regueiro BJ. Epidemiología y manifestaciones clínicas de las hepatitis virales. Enferm Infecc Microbiol Clin 2006;24(4):264-76

4. Instituto Nacional de Estadística. Extranjeros en la UE y en España. Cifras INE. Boletín informativo del Instituto Nacional de Estadística 2012; 6. Documento electrónico. [consultado 1 febrero 2014]. Disponible en: http://www.ine.es/ss/Satellite?L=es_ES\&c=INECifrasINE_C\&cid $=1259938022122 \& p=1254735116$ $567 \&$ pagename $=$ Productos YServicios\%2FPYSLayout

5. Esteban-Vasallo MD, Domínguez-Berjón MF, Astray-Mochales J, Gènova-Maleras R, Pérez-Sania A, Sánchez-Perruca L, et al. Prevalencia de enfermedades crónicas diagnosticadas en población inmigrante y autóctona. Gac Sanit 2009; 23: 548-52.
6. García-Fulgueiras A, García-Pina R, Morant C, de Larrea-Baz NF, Alvarez E. Burden of disease related to hepatitis $\mathrm{C}$ and hepatitis $\mathrm{B}$ in Spain: a methodological challenge of an unfolding health problem. J Viral Hepat 2011; 18: e453-60.

7. Valderas JM, Mendivila J, Parada A. Construcción de un filtro geográfico para la identificación en PubMed de estudios realizados en España. Rev Esp Cardiol. 2006; 59:1244-51

8. Intenational Organization for Migration (OIM); Internacional Migration Law, Glossary on Migration; OIM,Ginebra, 2004: p. 40. Disponible en: http://publications.iom.int/bookstore/free/IML_1_EN.pdf

9. Huerga Aramburu H, López-Vélez R. Estudio comparative de la patología infecciosa en niños inmigrantes de distintas procedencias. An Pediatr (Barc). 2004; 60: 1621.

10. Santiago B, Blázquez D, López G, Sainz T, Muñoz M, Alonso T, Moro M. Perfil serológico en gestantes extranjeras frente a VIH, VHB, VHC, virus de la rubéola, Toxoplasma gondii, Treponema pallidum, y Trypanosoma cruzi. Enferm Infecc Microbiol Clin. 2012; 30: 64-9.

11. Sampedro A, Mazuelas P, Rodríguez-Granger J, Torres E, Puertas A, Navarro JM. Marcadores serológicos en gestantes inmigrantes y autóctonas en Granada. Enferm Infecc Microbiol Clin. 2010; 28: 694-7.

12. Gutiérrez M, Tajada P, Alvarez A, De Julián R, Baquero M, Soriano V, et al. Prevalence of HIV-1 non-B subtypes, syphilis, HTLV, and hepatitis B and C viruses among immigrant sex workers in Madrid, Spain. J Med Virol. 2004; 74: 521-7.

13. Belza MJ, Clavo P, Ballesteros J, Menéndez B, Castilla J, Sanz S, et al. Condiciones laborales, conductas de riesgo y prevalencia de infecciones de transmisión sexual en mujeres inmigrantes que ejercen la prostitución en Madrid. Gac Sanit. 2004; 18: 177-83.

14. López-Vélez R, Huerga H, Turrientes MC. Infectious diseases in immigrants from the perspective of a tropical medicine referral unit. Am J Trop Med Hyg. 2003; 69 : 115-21.

15. Manzardo C, Treviño B, Gómez i Prat J, Cabezos J, Monguí E, Clavería I, et al. Communicable diseases in the immigrant population attended to in a tropical medicine unit: epidemiological aspects and public health issues. Travel Med Infect Dis. 2008; 6: 4-11.

16. Pérez-Molina JA, López-Vélez R, Navarro M, PérezElías MJ, Moreno S. Clinicoepidemiological characteristics of HIV-infected immigrants attended at a tropical medicine referral unit. J Travel Med. 2009; 16: 248-52. 
17. Monge-Maillo B, Jiménez BC, Pérez-Molina JA, Norman F, Navarro M, Pérez-Ayala A, et al. Imported infectious diseases in mobile populations, Spain. Emerg Infect Dis. 2009; 15: 1745-52.

18. Salas J, Vázquez J, Cabezas T, Lozano AB, Cabeza I. Infección por virus de hepatitis B (VHB) en inmigrantes subsaharianos en Almería. Enferm Infecc Microbiol Clin. 2011; 29: 121-3.

19. Pérez-Molina JA, Herrero-Martínez JM, Norman F, Pérez-Ayala A, Monge-Mahillo B, Navarro-Beltrá $\mathrm{M}$, et al. Clinical, epidemiological characteristics and indications for liver biopsy and treatment in immigrants with chronic hepatitis B at a referral hospital in Madrid. J Viral Hepat. 2011; 18: 294-9.

20. Vall Mayans M, Arellano E, Armengol P, Escribà JM, Loureiro E, Saladié P, et al. Infección por el virus de la inmunodeficiencia humana y otras infecciones de transmisión sexual en inmigrantes de Barcelona. Enferm Infecc Microbiol Clin. 2002; 20: 154-6.

21. Toro C, Jiménez V, Rodríguez C, Del Romero J, Rodés B, Holguín A, et al. Molecular and epidemiological characteristics of blood-borne virus infections among recent immigrants in Spain. J Med Virol. 2006; 78:1599-608

22. Hernando Jerez A, García-Cerrada C, Ortega FP, García RR, Gómez RH, González CC, et al. Infección por el VIH en inmigrantes: diferencias clínico-epidemiológicas con la población autóctona en un área de salud de la Comunidad de Madrid (2002-2004). Enferm Infecc Microbiol Clin. 2007; 25: 441-5.

23. Caro-Murillo AM, Gutiérrez F, Manuel Ramos J, Sobrino P, Miró JM, López-Cortés LF, Tural C, Moreno A, de Los Santos I, Murillas J, Camino X, Salavert M, Rubio R, Moreno S, del Amo J; CoRIS. Infección por virus de la inmunodeficiencia humana en inmigrantes en España: características epidemiológicas y presentación clínica en la cohorte CoRIS, 2004-2006. Enferm Infecc Microbiol Clin. 2009; 27: 380-8.

24. Bottecchia M, Madejón A, Puente S, García-Samaniego J, Rivas P, Herrero D, et al. Detection of hepatitis B virus genotype A3 and primary drug resistance mutations in African immigrants with chronic hepatitis B in Spain. J Antimicrob Chemother. 2011; 66: 641-4.

25. Ramos JM, Masiá M, Padilla S, Escolano C, Bernal E, Gutiérrez F. Enfermedades importadas y no importadas en la población inmigrante. Una década de experiencia desde una unidad de enfermedades infecciosas. Enferm Infecc Microbiol Clin. 2011; 29: 185 92.
26. Valerio L, Barro S, Pérez B, Roca C, Fernández J, Solsona L, Aguilar A, Escribà JM. Seroprevalencia de marcadores de hepatitis crónica vírica en 791 inmigrantes recientes en Cataluña, España. Recomendaciones de cribado y vacunación contra la hepatitis B. Rev Clin Esp. 2008; 208: 426-31.

27. Ramos JM, Pastor C, Masía MM, Cascales E, Royo G, Gutiérrez-Rodero F. Examen de salud en la población inmigrante: prevalencia de infección tuberculosa latente, hepatitis $\mathrm{B}$, hepatitis $\mathrm{C}$, infección por el VIH y sífilis. Enferm Infecc Microbiol Clin. 2003; 21: 540-2.

28. Ramos JM, Milla A, Rodríguez JC, Gutiérrez F. Seroprevalencia frente a Toxoplasma gondii, virus de la rubéola, virus de la hepatitis B, VIH y sífilis en gestantes extranjeras en Elche y comarca. Med Clin (Barc). 2007; 129: 677-8.

29. Salleras L, Domínguez A, Bruguera M, Plans P, Espuñes J, Costa J, et al. Seroepidemiology of hepatitis B virus infection in pregnant women in Catalonia (Spain). J Clin Virol. 2009; 44: 329-32.

30. Guerrero Espejo A, Colomina Rodríguez J. Población inmigrante y enfermedades infecciosas. Rev Esp Salud Pubica 2004; 78: 565-70.

31. Haj-Ali Saflo O, Miguel Hernández Guijo J. Coste-eficacia del tratamiento de la hepatitis $\mathrm{C}$ crónica en España. Gastroenterol Hepatol. 2009; 32: 472-482.

32. Blázquez-Pérez A, San Miguel R, Mar J. Cost-Effectiveness Analysis of Triple Therapy with Protease Inhibitors in Treatment-Naive Hepatitis C Patients. PharmacoEconomics 2013; 31: 919-931.

33. Turnes J, Romero-Gómez M, Planas R, Solà R, García-Samaniego J, Diago M, et al. Pharmacoeconomic analysis of the treatment of chronic hepatitis $\mathrm{C}$ with peginterferon alfa- $2 a$ or peginterferon alfa- $2 b$ plus ribavirin in Spain. Gastroenterol Hepatol. 2013; 36: 555-64.

34. Poves-Martínez E, del Pozo-Prieto D, CosteroPastor B, Borrego-Rodríguez G, Beceiro-Pedroño I, Sanz-García C, et al. Diagnostic incidence of the presence of positive HBsAg: epidemiologic, clinical, and virological characteristics. Rev Esp Enferm Dig. 2012; 104: 10-5. 